areas are almost superimposed, and their shape gives a clue to the shape of the pupil (or coloboma) (Fig. 137).

3. Anterior synechiae.-An adhesion of one margin of the pupil to the cornea will give two patches of light on the cornea, having one common border, corresponding to the synechia, whereas the free margin of the pupil allows a separation between the other margins of the illuminated patches (Fig. $13^{8}$ ).

4. Alteration in the size of the pupil by mydriatics or miotics can be observed and recorded, and the presence of holes in the iris, or an atrophic thin iris frequently can be determined.

The examples given will serve to show the usefulness of this method of examination in cases of this type, and the information gained has proved of real service in dealing with cases of opaque corneae in man, and their treatment by corneal transplantation.

\title{
SUPPLEMENTARY NOTE ON \\ IRIS INCLUSION FOR CHRONIC GLAUCOMA \\ BY
}

\section{H. HERBERT}

THE recent Oxford Congress debate on the operative treatment of glaucoma seems to provide an occasion for the remedy of faults and omissions in my paper "The Future of Iris Inclusion in Glaucoma,” Brit. Jl. Ophthal., Vol. XIV, pp. 433-48, 1930.

\section{I. - The later phases in Operative Development}

In the above paper the thesis. was developed that progress in iridencleisis had for more than a decade resolved itself into a practical study of conjunctival fibrosis, protective against late infection. Work begun in India during the War, and continued fairly steadily up to the date of the paper, was reviewed.

There were three phases in this experience. The first was that of the uncut iris prolapse, condemned chiefly on account of a remote liability to the formation of a most dangerous leaking point in an over-stretched prolapse undergoing fibrosis.

Throughout the long second period the essential feature was free subconjunctival radial division of the iris with straight, narrow, blunt-pointed scissors, close to the forceps gripping the iris. By this means, together with such mydriasis as was obtainable by the omission of miotics from the previous afternoon or evening, and by 
the free use of cocaine with adrenalin and atropine discs before operation, a degree of uniformity in results was obtained. Permanent relief of tension was assured, together with the desired restriction in the size and elevation of the prolapse, and a variable degree of fibrosis.

The final period was a very short one, and on that account, perhaps, to be reckoned one of great promise rather than of assured fulfilment. There was a very definite increase in fibrosis secured without any restriction of the required subconjunctival filtration.

This more assured fibrosis was obtained simply by the addition of a measured iridodialysis to the operative technique. The iris, seized near its base, was detached to the extent of $3.4 \mathrm{~mm}$. by a steady pull of the iris forceps towards the centre of the pupil. Since writing the paper above mentioned I have regretted that in it I omitted to define the size of the iridodialysis utilized. This was due to limited experience. But obviously a very small detachment might have little or no effect. On the other hand, I believe that an unnecessarily large detachment could do no serious harm. It would bring merely the defects of any abnormally large and distorted pupil.

The experience still calls for wide general confirmation and exploitation. I have little doubt that this need will be met. Some work is being done on these lines now.

Clinical observation has shown clearly that the new tissue is of purely conjunctival origin, extending down into the underlying displaced iris, largely replacing it.

There is the wide area of dilated tortuous conjunctival bloodvessels. At first it seemed reasonable to attribute both the abnormal vascularity and the new fibrous tissue largely to friction and pressure of the upper lid upon the tissues, elevated by leaking aqueous. It is now clear, however, that the essential proliferative stimulus comes from mildly irritative products of breaking-down displaced iris tissue - products imprisoned for a time beneath the conjunctiva.

This is shown by the absolute localization of the new tissue to the site of the prolapse, by its very definite incorporation with the prolapse, and by the increased certainty and degree of the formative response that has resulted, in the final developmental phase, obviously from the enhanced rapidity and completeness of the degenerative changes in the detached and displaced iris.

It is shown also by the very rapid shrinkage of the abnormal conjunctival blood-vessels as the small patch of new fibrous tissue becomes visible.

The key to the degenerative changes in the iris prolapse has been found in clinical observation of the changes that have taken place within the eye in rare instances of old unintended operative iridodialysis. 
A separated strip of iris may have been left at some operation almost in its normal position, normally attached only at one end, the other (displaced) end lying perhaps impacted in a sclero-corneal scar-line. The detached strip of iris is eventually reduced to a rather narrow grey band. The changes comprise shrinkage, loss of pigment and more or less loss of elasticity and contractility. In spite of the loss of colour there is no suggestion whatever of any new (fibrous) tissue in the shrunken iris.

The breaking down of spongy iris tissue has been accomplished quietly, and the products carried away suspended in the aqueous.

Some degree of these degenerative changes of detached iris must take place in any portion of iris permanently displaced under the conjunctiva. This was shown in early days after iris-inclusion operations of various kinds on the deeply pigmented Indian eyes, by a moderate permanent accumulation of black pigment granules beneath the conjunctiva immediately surrounding the prolapse.

And it is inevitable that in the final (third) operative period of prolapse with detachment of the iris-base, the breaking down of iris cells must have been more complete and more certain. The practical ultimate result must have been that there was little or no essentially uveal tissue left in the subconjunctival nodule, beyond the attenuated lining of pigment epithelium. (See photo-micrographs by Verhoeff and by Holth referred to in my previous paper.) That is to say, beyond the epithelial lining practically the only true uveal tissues remaining must have lain exclusively within the globe.

Contact of the covering conjunctiva with the degenerating iris nodule is essential if new tissue is to be formed. This was shown in early days by the complete absence of fibrosis in cases where a small prolapse lay buried beneath oedematous conjunctiva.

Contact alone, without any lid pressure, may be fully effective. This has been shown where, after previous operation of some kind, the conjunctiva has been so tied down locally to the sclera, that the small iris-nodule formed in the glaucoma treatment has produced a sharply defined elevation, distinctly above the level of the surrounding conjunctiva.

The sides of the small iris loop may then have a conjunctival covering as closely applied as that over the summit. The result is that at the sides, where there is no pressure and no friction of the lid, the fibrosis is as well developed as on top of the elevation.

One might fear that in such cases subconjunctival filtration might be insufficient for the relief of the glaucoma. But this has not been the case, apparently because there must always be a gap between a conjunctival product and the underlying sclerotic.

The fibrosis being essentially superficial in origin-conjunctival, and essentially of temporary growth-ending with the degenerative 
changes in the displaced iris tissue, it can never be so excessive as to obstruct subconjunctival drainage.

According to present prospects, in this form of treatment there can be neither late infection nor late return of tension, and the filtration is never markedly excessive. In dealing with the spongy iris tissue there seems to be more natural adaptation throughout to the needs of individual cases, than with iris-free drainage.

The important question arises: Is the early increased fibrosis secured by the addition of iridodialysis to the operative technique likely to be always a permanent acquisition? There is some reason to hope so.

I have quite recently come across a patient upon whose eyes I operated by iridencleisis eleven years ago. In one eye there was unintentional iridodialysis; the detachment is unpleasantly obvious now through the cornea. In this eye there is no uveal pigment visible anywhere under the conjunctiva, and the site of the original prolapse is indicated by an ill-defined area of subconjunctival fibrous tissue. The whole filtration effect must be classed as satisfactory.

To contrast with the above, the result in the fellow eye, in which there was no iris detachment, is strongly objectionable. According to the new idea, the blood supply of displaced subconjunctival iris was not sufficiently interfered with. The early degenerative changes in the iris loop were not sufficiently massive, immediate and concentrated, to stimulate the full available proliferative response from the covering conjunctiva. About a year after the operation a note was made, "complete fibrosis of part of prolapsed iris. Dark patches showing incomplete fibrosis."

Since then there has been some slow absorption of the unfibrosed, abnormally placed, iris tissue. Now, situated about the middle of the widespread conjunctival oedema there is a small defined absolutely black patch, bulging slightly-evidently pigment epithelium, covered by thin transparent conjunctival epithelium.

If such a result were not surely preventable no one could recommend or practise iridencleisis.

\section{II.-Early Sympathetic Ophthalmitis}

The fear of sympathetic disease is still, I suppose, the great deterrent in the question of treatment by iris-inclusion. It remains only to exclude the possibility of the seeds of the disease being sown during operation or in the first few days afterwards. On this account it is worth while to recall my only post-operative case of sympathetic ophthalmitis in this country. The case was recorded in the 1930 paper, but it could not be dealt with satisfactorily in a paper that was otherwise becoming too long for sustained interest.

It was most fortunate from the point of view of iris-inclusion 
treatment that there was no trace of iris impaction in the case, which was one of ordinary combined cataract extraction. The only early trouble was that, owing to a combination of unusual circumstances, the usual 2 minutes' perchoride of mercury irrigation (1 in 3000 ) of the conjunctiva, before the cocaine instillations, had been forgotten. And the omission was not detected till the instillations had all been made. It was impossible then to secure the usual effect of the pre-cocaine irrigation - some shedding of epithelial cells, embedded in mucus, to be washed away in sterile water.

It seemed desirable also to insure doubly against remote dangers, because it had been arranged that I was to see the patient once only in the after-treatment, namely, on the following day. For these two reasons I irrigated for three minutes exactly, instead of the usual two minutes, though the conjunctiva was perfectly healthy, pale and smooth. This period was slightly longer than I have used in any - other case continuously, though in the case of slightly unhealthylooking conjunctivae the routine two-minutes' pre-cocaine irrigation was often extended a little, and (or) supplemented by a short additional application immediately before operation.

This exceptional treatment proved to be a great mistake. It seems reasonably certain that the operation would have been perfectly successful, if there had been no conjunctival treatment at all.

On the next day the upper lid was found to be glued down so firmly to the cornea that it resisted mild attempts to separate it. Other similar adhesions that I have seen have given way easily to a little manipulation, and no harm has ever resulted. As there was no cocaine at hand I left the lid adherent. This also turned out to be a mistake. I ought to have persisted in freeing the lid. Though on the following day, I understand, the lid had separated naturally, the pull of the adhesion had been sufficient to produce a permanent gaping of the sclero-corneal section.

A variable slight separation of the deep wound margins is quite usual when a complete conjunctival flap has been utilized. Possibly this may be more certain where, as in this case, there has been a little preliminary sliding of the conjunctiva on the point of the knife in making the incision. But in this case the separation was quite exceptional, producing an obvious astigmatic distortion of the neighbouring cornea, sufficient to prove clearly that the gaping was, in part, due to the pull of the adherent upper lid on the cornea.

A flaccid lid, of course, could exert no pull on an eye at rest in its normal position. The drag could be exerted only when the orbicularis muscle contracted, or when the eyeball moved. That is, the traction must have been intermittent and active, spasmodic, producing some degree of suction at times. Any conjunctival contents lying close to the wound would be readily drawn into the 
eye, especially considering that the conjunctival flap also was almost certainly adherent to the lid, so that the conjunctival wound would necessarily be pulled open.

Thus, to summarize: A suction mechanism was present for a period unique in my experience-more than twenty-four hours. A practically unique degree of corneal distortion sufficed to show that the mechanism had actually worked. As a not unnatural result of such working, indrawing conjunctival contents, we have the sympathetic disease, which was also unique to the degree above stated. That the association of such quite exceptional features was one of cause and effect is further borne out by the somewhat exceptional course of the disease.

Corresponding with the absence of any iris incarceration in the wound, the pupil and iridectomy-gap, though filled with organizing exudate, remained widely dilated to the end. They were fully dilated by the usual routine atropine instillations after the operation, and remained so under ordinary treatment.

There was no other possible suggestion in the case - nothing else in the least imperfect or unusual. The presumption of the working of a conjunctival micro-organism was very strong-I think, conclusive.

Though the case stands as an extreme warning against excessive, and more particularly against ill-timed, use of the antiseptic solution, it should not be allowed to detract at all from the undoubted value of its correct employment. This employment in India did serve almost completely to exclude both sympathetic and septic infections.

The question was dealt with in the 1930 paper. On present evidence practical temporary sterilization of the conjunctival surface, obtainable by several methods, affords ample security for any correctly planned iridencleisis operation. Consequently, in operating without one of these proved safeguards, one unnecessarily incurs a very grave responsibility.

To consider for a moment the active agent in the disease: It being practically certain in this case that the specific organism was drawn into the eye from the conjunctiva by the mechanism portrayed, valuable material for research was wasted. A somewhat exceptional opportunity was thrown away.

"There is no originality of thought in incriminating a virus without supporting evidence" (R. T. Brain, Brit. Med. Jl., p. 194, July 29, 1933). But, the search for bacteria in this disease having failed, some organization appears to be needed for collecting a little of the very scanty available material, and arranging for its investigation at a laboratory, such as the Freedom Research Laboratory, London Hospital, where virus research work is being carried on.

In this case the conjunctival epithelium might have shown 
inclusion bodies and virus particles. A virus might have been grown on epithelial scrapings, and possibly the strain maintained for exhaustive research. From what is known of viruses, is it not much more likely that this perfectly normal conjunctiva should have served as a carrier for a virus than for a pathogenic bacterium ?

P.S.-Neither in the above, nor in the 1930 paper, have I emphasized sufficiently, as I ought to have done, my firm conviction that the new fibrotic development will prove to be a far-reaching discovery, bound to influence greatly the future treatment of chronic glaucoma.

2. The more recent history of an extraordinary case discussed on pp. $447-8$ of the Brit. Jl. of Ophthal., Vol. XIV, 1930, supplies a warning that durable conjunctival fibrosis may under exceptional conditions be unobtainable by iridencleisis in the case of an eye that has already been repeatedly operated upon by other methods unsuccessfully.

In this particular eye four small flap sclerectomies had failed to relieve the plus tension, though at the subsequent iridencleisis the old scleral incisions (still visible) were proved to have remained open. The only possible explanation of the invariable failure of the sclerectomies was an abnormal restriction of subconjunctival diffusion and absorption of leaking aqueous - a restriction due to post-operative fibrinous deposits. A reasonable explanation of the formation of these very exceptional deposits was given.

After the iridencleisis, by which the tension was finally relieved, there was most promising early fibrosis of the prolapsed nodule of iris. But now this new fibrous tissue has all disappeared, and in place of the elevated nodule there is a wider flat defined patch of sodden semi-vesicular conjunctiva-a condition with which we have been too familiar. The abnormally restricted subconjunctival diffusion of aqueous must be held directly responsible for the objectionable leaking patch of conjunctiva.

H. H. 